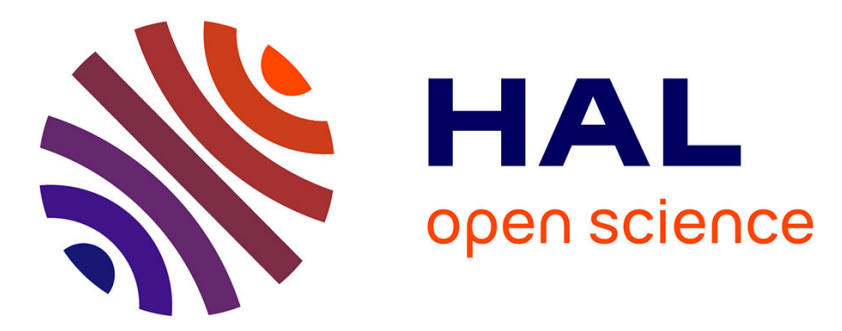

\title{
Dispositif pour l'étude par réflexion sélective des raies spectrales de vapeurs à haute pression et haute température. Application à la vapeur de mercure
}

\author{
P. Laporte, A. Leycuras
}

\section{- To cite this version:}

P. Laporte, A. Leycuras. Dispositif pour l'étude par réflexion sélective des raies spectrales de vapeurs à haute pression et haute température. Application à la vapeur de mercure. Revue de Physique Appliquée, 1972, 7 (3), pp.193-196. 10.1051/rphysap:0197200703019300 . jpa-00243618

\section{HAL Id: jpa-00243618 https://hal.science/jpa-00243618}

Submitted on 1 Jan 1972

HAL is a multi-disciplinary open access archive for the deposit and dissemination of scientific research documents, whether they are published or not. The documents may come from teaching and research institutions in France or abroad, or from public or private research centers.
L'archive ouverte pluridisciplinaire HAL, est destinée au dépôt et à la diffusion de documents scientifiques de niveau recherche, publiés ou non, émanant des établissements d'enseignement et de recherche français ou étrangers, des laboratoires publics ou privés. 


\title{
DISPOSITIF POUR L'ÉTUDE PAR RÉFLEXION SÉLECTIVE DES RAIES SPECTRALES DE VAPEURS A HAUTE PRESSION ET HAUTE TEMPÉRATURE. APPLICATION A LA VAPEUR DE MERCURE
}

\author{
P. LAPORTE et A. LEYCURAS \\ Laboratoire des Hautes Pressions \\ Centre National de la Recherche Scientifique, 92-Bellevue
}

(Reçu le 21 février 1972)

\begin{abstract}
Résumé. - On décrit un appareillage haute pression haute température destiné à l'étude de la réflexion sélective d'une vapeur métallique, et plus particulièrement de la vapeur de mercure pour laquelle le domaine de pression s'étend de 1 à 500 bar et le domaine de température de 350 à $1200^{\circ} \mathrm{C}$

Abstract. - A high pressure high temperature apparatus for selective reflection studies of metallic vapors is described. Results on mercury vapor in the pressure range 1-500 bar and temperature range $350-1200^{\circ} \mathrm{C}$ are presented.
\end{abstract}

I. Introduction. Les vapeurs métalliques pures sous pression n'ont fait l'objet dans le passé que de très rares études. Toutefois, de telles études sont en net développement depuis quelques années malgré les difficultés expérimentales soulevées.

Les travaux publiés portent principalement sur les propriétés de transport (par exemple [1], [2], [3], [4]) et rares sont celles qui portent sur les propriétés optiques [5], [6]. Pourtant ces dernières propriétés, et leur évolution en fonction des conditions physiques, sont susceptibles d'apporter des informations, tant sur les interactions que sur l'association en molécules, et éventuellement, à haute densité sur le mécanisme du passage isolant - semi-conducteur-métal.

Une étude en réflexion devient nécessaire chaque fois que l'on veut étudier une bande de forte absorption. Dans ce cas, une cuve en transmission devrait en effet être très mince (ordre de grandeur $1 \mu$ ), ce qui est difficilement réalisable dans le domaine de température à atteindre. C'est dans des domaines spectraux, où existe une raie de résonance atomique, que le phénomène de réflexion devient particulièrement intéressant. On observe alors un maximum du facteur de réflexion (réflexion sélective de Wood [7]).

Le mercure présente l'avantage de posséder le point critique le plus bas de tous les métaux (1500 b, $1500^{\circ} \mathrm{C}$ ) [2], [3], [8], ce qui permet d'obtenir des densités élevées sans monter très haut en température. De plus, il ne pose pas de problème trop délicat de corrosion chimique à chaud.

L'étude de la réflexion sélective de la raie de résonance $2537 \AA$ de la vapeur de mercure avait déjà été abordée par Welsh et coll. [5]-[9] dans les domaines 0-8 bar et 41-345 bar par deux approches techniques différentes. Cependant l'importante discontinuité entre les résultats des deux séries de mesures ainsi qu'une certaine dispersion dans les résultats de la deuxième série (visibles sur la figure 5) nous ont incités à reprendre cette étude et à entreprendre la réalisation d'un unique ensemble expérimental permettant d'étudier un domaine de densité d'une part plus vaste et d'autre part sans interruption.

Le dispositif que nous décrivons a permis de suivre de façon continue le phénomène dans le cas du mercure pur pour des densités comprises entre $0,01 \mathrm{~g} / \mathrm{cm}^{3}$ et $0,9 \mathrm{~g} / \mathrm{cm}^{3}$; ceci correspond aux conditions 350 $1200^{\circ} \mathrm{C}$ et 1 à 500 bar. C'est à notre connaissance la seule étude de ce type qui ait été réalisée jusqu'à des densités aussi élevées.

La mise au point de l'ensemble que nous allons décrire maintenant en détail nous a montré la très grande rigueur avec laquelle il devait être réalisé pour obtenir une bonne continuité et reproductibilité des résultats, conditions essentielles pour pouvoir interpréter ceux-ci.

II. Dispositif expérimental. - Le but de l'expérience envisagée est de recueillir la lumière réfléchie par un dioptre $\mathrm{R}$ (solide transparent-vapeur de mercure) et d'en mesurer l'intensité en fonction de la longueur d'onde. Mais les conditions de température et de pression d'une part, et des phénomènes optiques parasites d'autre part, rendent cette mesure délicate.

C'est l'analyse de ces différents facteurs qui justifie la disposition des éléments de l'appareillage ainsi que le choix des solutions techniques adoptées.

Les conditions de température et de pression conduisent à adopter une enceinte de pression à chauffage interne afin d'éviter des contraintes mécaniques sur certaines pièces optiques portées à température élevée ; elles sont ainsi en équipression et les efforts exercés par la pression sont supportés par des pièces mécaniques à température normale.

La température élevée de la cellule renfermant la 
vapəur oblige à la placer avant l'appareil dispersif, ceci afin de soustraire le récepteur à la totalité du rayonnement des pièces chaudes.

Le domaine relativement étroit de longueur d'onde étudié se prêtait bien à l'emploi d'une réception photoélectrique utilisant un monochromateur qui comporte un certain nombre d'avantages tels que l'obtention rapide des résultats et la possibilité, à longueur d'onde constante, de connaître l'évolution des phénomènes (changement de phase, instabilité...). Ce contrôle permanent a permis de préciser le meilleur mode opératoire en fonction du domaine étudié comme nous le verrons plus loin.

On est ainsi conduit à la disposition représentée schématiquement sur la figure 1 où l'on peut suivre le trajet des faisceaux lumineux. Nous ne décrirons en détail que les éléments les plus importants.

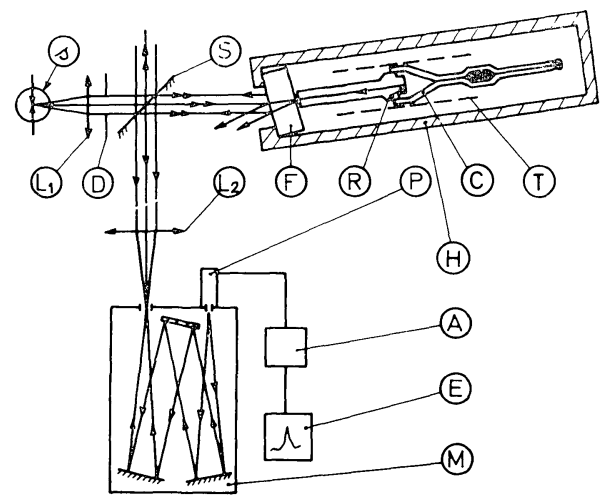

FIG. 1. - Dispositif expérimental : s, Source ; S, Séparatrice ; $\mathbf{L}_{1}, \mathbf{L}_{2}$, Lentilles ; D, diaphragme ; F, Fenêtre haute pression ; $\mathrm{R}$, Dioptre solide vapeur; P, Photomultiplicateur; C, Cellule ; $\mathrm{T}$, Enroulement chauffant; H, Corps d'enceinte haute pression; A, Amplificateur à courant continu ; E, Enregistreur ; M, Monochromateur.

1. EnSEMble haute PRESSiON. - L'enceinte utilisée a été précédemment décrite [10]. La figure 2 montre la disposition des éléments internes ainsi que le conduit conique de lumière qui a dû être aménagé pour nos expériences en incidence oblique par rapport à la fenêtre d'entrée.

Afin de préserver la pureté du gaz compresseur (Argon), nous avons utilisé un séparateur à membrane métallique qui a été spécialement mis au point au laboratoire pour ce type d'application [11]. Le compresseur est un compresseur à huile classique Sprague.

Trois manomètres Heise-Bourdon (précision $1 / 1000^{\mathrm{e}}$ de la pleine échelle) permettent la mesure de la pression dans tout domaine exploré.

2. Ensemble DE ChAuffage. - La réalisation de fours sous pression demande toujours un soin particulier en raison de l'influence très importante des courants de convection [12]. Le four que nous avons réalisé est à enroulement chauffant de platine et à isolement mixte, écrans métalliques et alumine (Fig. 2). Les écrans métalliques sont constitués d'une feuille mince de nickel piquetée et bobinée. On obtient

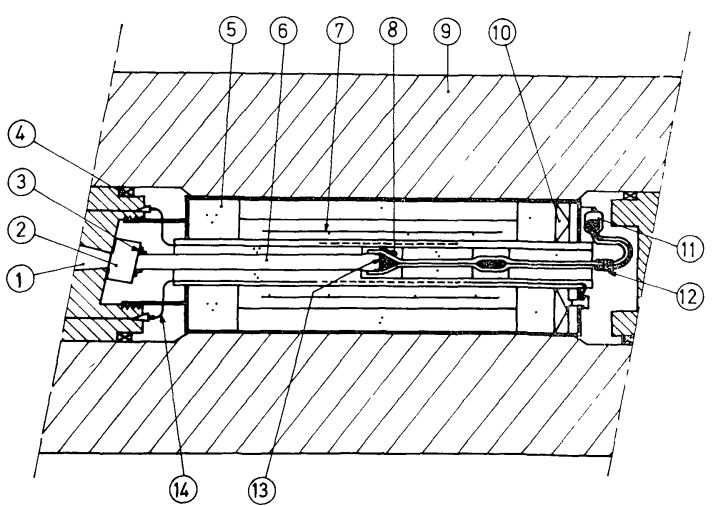

FIG. 2. - Ensemble four-cellule : 1. Entrée optique ; 2. Fenêtre de pression. - 3. Gaine d'or; 4. Joints; 5. $\left(*_{*}^{*}\right)$ Alumines; 6. Cellule ; 7. Ecrans métalliques ; 8. Thermocouple ; 9. Corps de l'enceinte haute pression; 10. Ressort; 11. Réservoir de mercure; 12. Gaine Téflon; 13. Cavité pour la vapeur; 14. Entrée du courant de chauffage.

ainsi environ 8 couches par mm. Cette technique [13] assure des gradients de l'ordre de $100^{\circ} \mathrm{C}$ par mm, elle conduit donc à d'excellents rendements thermiques (dissipation toujours inférieure à $600 \mathrm{~W}$ pour des expériences décrites ici, soit une puissance de $6 \mathrm{~W} / \mathrm{cm}^{2}$ rayonnée par le ruban de chauffage).

L'enroulement chauffant est alimenté soit par une chaîne de régulation classique, soit par un ensemble stabilisateur de tension - rhéotor - transformateur d'isolement, dispositif qui s'est avéré suffisant.

La température est mesurée au moyen d'un thermocouple platine-platine rhodié $10 \%$ placé au contact de la cellule au niveau de la zone de réflexion. Un ensemble de mesures avec fils compensés et source froide assure une précision meilleure que le degré au point de fusion de l'or, qui nous a servi de contrôle.

3. Optique. - Nous donnerons quelques précisions n'apparaissant pas sur la figure 1: la source, assez ponctuelle, est une lampe à deutérium qui émet un spectre continu dans l'ultraviolet. Le faisceau lumineux tombe en incidence normale sur le dioptre (R). L'ensemble des pièces optiques est réalisé en silice fondue (Suprasil), en particulier la séparatrice, semiréfléchissante, est constituée par une lame de silice recouverte d'un dépôt mince d'aluminium.

Nous avons utilisé un monochromateur à réseau en montage Czerny-Turner, et construit par la société Jobin-Yvon, susceptible de donner une résolution maximale de 150000 en simple passage. Il est ouvert à $\mathrm{F} / 12$.

La réception est tout à fait classique et comporte un photo-multiplicateur de très bonne qualité ( $\mathrm{R} 106$ de HTV à enveloppe de quartz, sélectionné), un amplificateur à courant continu et un enregistreur. La parfaite linéarité de réponse de l'ensemble a été contrôlée à l'aide de grilles étalonnées.

L'essentiel de la mise au point consiste à réaliser une cellule permettant d'éliminer tous les facteurs parasites. Celle-ci est d'une seule pièce de silice, elle apparaît sur les figures 1 et 2 . Elle comporte : un 
barreau massif soudé à un tube conique limitant une cavité où le mercure est vaporisé, un capillaire de communication, un réservoir de préchauffage, puis un vase d'expansion froid qui assure une très légère surpression dans la cellule. L'ensemble est donc pratiquement en équipression dans l'enceinte.

La face d'entrée de la cellule est au contact de la fenêtre de pression, ce qui évite toute perturbation du faisceau liée à la convection et toute absorption sélective due à la présence éventuelle de traces de mercure dans l'enceinte. Par précaution supplémentaire une gaine d'or [3] figure 2 est introduite en guise de piège à mercure. L'enregistrement du pouvoir réflecteur du mercure liquide (non sélectif) dans la région $2537 \AA$ en début d'expérience, puis à chaud et sous pression, permet de s'assurer de l'absence d'absorption sélective.

Cette cellule présente une bonne transmission UV à haute température. Le matériau utilisé et le réservoir de préchauffage intermédiaire lui confèrent une bonne résistance aux chocs thermiques en cas de recondensations à haute température. La forme conique de la cavité [13] et le biseau que présente la face d'entrée ont pour but d'écarter de l'axe d'observation toute réflexion autre que celle provenant $d u$ dioptre étudié. L'angle du biseau $\left(2^{\circ}\right)$ est faible et ne risque pas de provoquer une déconjugaison de l'ensemble dans un domaine spectral limité.

Un calcul simple montre de plus que, avec l'optique choisie, la lumière diffusée est toujours négligeable vis-à-vis de la lumière réfléchie par le dioptre quartz mercure.

III. Conduite des expériences. - 1 . OBTENTION DE LA VAPEUR SOUS PRESSION $P$ ET TEMPÉRATURE $T$. - Le mercure, de très haute pureté $(7 \mathrm{~N})$, est introduit sous vide dans la cellule puis on procède au montage de l'ensemble expérimental. Selon le domaine de pression, le mode opératoire diffère :

a) Aux moyennes densités, où l'on risque des instabilités en cours d'ébullition, on opère de la façon suivante : vaporisation à la pression atmosphérique, puis surchauffe de la vapeur juśqu'à la température recherchée, enfin compression jusqu'à la pression désirée : l'enregistrement optique n'est fait qu'une fois obtenue la stabilisation et une série d'expériences peut être menée sans recondensation.

b) Aux densités élevées, les ébullitions sont stables et on peut agir sur la température ou sur la pression indépendamment. Dans des expériences prolongées à $1200^{\circ} \mathrm{C}$ la partie chaude de la cellule est détruite lors de la redescente en température, en raison d'un changement d'état de la silice.

Des recondensations effectuées volontairement en cours d'expérience ou la recondensation finale à la fin d'une série permettent de s'assurer qu'il n'existait aucune trace de gaz étranger mélangée à la vapeur de mercure. De plus, l'ébullition en position horizontale du mercure contenu dans la cellule permet d'entraîner tout gaz dissous qui pourrait être libéré lors de la première montée en température.

2. Mesures optiQues. - En général, pour un point d'étude $(P, T)$ on réalise plusieurs enregistrements consécutifs dont l'un au moins comporte une référence en longueur d'onde. Celle-ci est obtenue de la façon suivante :

Lors du défilement, la position de la longueur d'onde $2536,5 \AA$, correspondant à la raie de résonance du mercure à basse densité, est connue par avance avec assez de précision. Une cuve contenant de la vapeur de mercure (basse densité) est introduite sur le parcours $\mathrm{du}$ faisceau pendant le bref instant correspondant au passage de $\lambda=2536,5 \AA$. On obtient ainsi un enregistrement perturbé seulement dans un faible domaine spectral. La comparaison de deux enregistrements consécutifs donne la référence souhaitée. Cette cuve permet également de contrôler la résolution de l'ensemble. Celle-ci a varié de $0,06 \AA$ à $0,2 \AA$ suivant les besoins de l'expérience.

En ce qui concerne l'étalonnage en intensité, il n'est pas possible de l'obtenir directement. Il est donc fourni sur chaque enregistrement par le signal obtenu dans les régions de transparence qui encadrent le phénomène. Le pouvoir réflecteur correspondant Ro du dioptre est alors sensiblement constant dans un domaine spectral peu étendu. On le calcule en reprenant l'analyse décrite par Welsh et coll. [5] mais en utilisant l'équation d'état de Sugawara [14] pour la vapeur de mercure pour $P<300$ bar et les résultats de Kikoin [2] pour $P>300$ bar.

IV. Résultats. - Pour illustrer les performances de l'appareillage nous présentons quelques résultats représentatifs dans le domaine $350-1200{ }^{\circ} \mathrm{C}$ et $1-500$ bar (Fig. 3 et 4 ). Ces résultats se sont avérés très reproductibles sur plusieurs séries d'expériences.

Les deux enregistrements présentés correspondent à un domaine de densité où aucune observation n'avait été réalisée auparavant. On peut remarquer la très

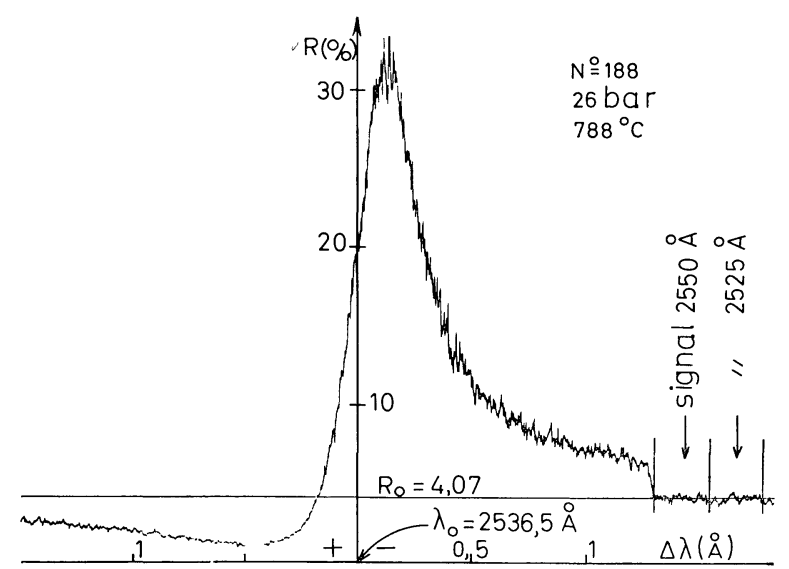

Fig. 3. - Spectre de réflexion du dioptre silice-vapeur de mercure (densité $0,060 \mathrm{~g} / \mathrm{cm}^{3}$ ) résolution $0,06 \AA$. 


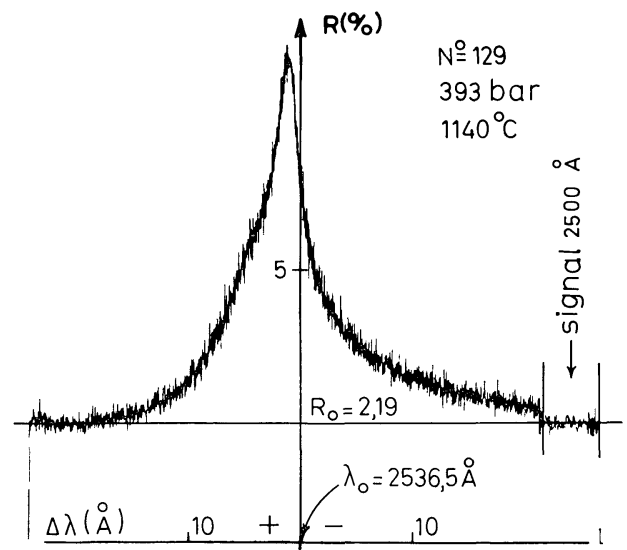

FIG. 4. - Spectre de réflexion du dioptre silice-vapeur de mercure (densité $0,76 \mathrm{~g} / \mathrm{cm}^{3}$ ) résolution $0,2 \AA$.

importante évolution du phénomène qui se caractérise par un élargissement, un déplacement du maximum, l'apparition à haute densité d'un épaulement, côté grandes longueurs d'onde (Fig. 4), ainsi qu'une variation importante de l'intensité du pouvoir réflecteur maximum. L'épaulement que l'on observe apparaît sous forme d'un maximum secondaire à densité moyenne. Il correspond à la bande 2540,5 Å observée à basse densité par différents auteurs [15]-[19] et qui semble caractéristique des couples $\mathrm{Hg}_{2}$.

Nous donnons sur la figure 5 l'évolution du pouvoir réflecteur maximum avec la densité. Celui-ci ne caractérise directement aucune grandeur physique, mais il est un indice de reproductibilité et de continuité des résultats. Nous avons porté également sur cette figure les points correspondant aux deux séries de mesures de Welsh et coll. [5]-[9]. A basse densité il y a bon accord entre les deux groupes d'observations, tandis que nous obtenons à haute densité des pouvoirs réflecteurs beaucoup plus élevés et une évolution régulière. Notons que les trois points les plus hauts en densité de Welsh ont été obtenus par une seule expérience chacun, tandis que les autres représentent la moyenne de plusieurs enregistrements. Il est important de remarquer que tous les facteurs parasites envisagés plus haut concourent à réduire le pouvoir réflecteur mesuré. Nous pensons que c'est

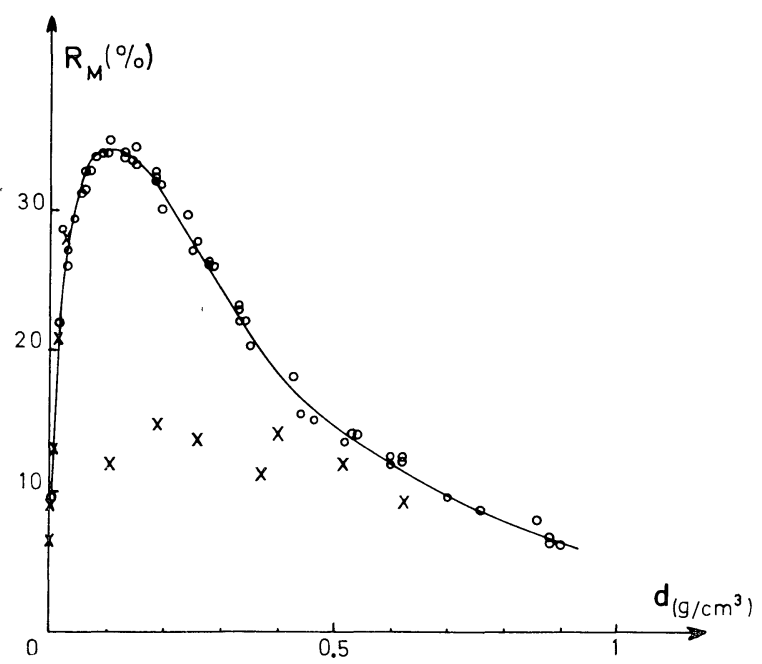

FIG. 5. - Evolution du pouvoir réflecteur maximum du dioptre silice-vapeur de mercure, en fonction de la densité de la vapeur, au vooisinage de la raie de résonance $2536,5 \AA$. $X=$ points correspondant aux observations de Welsh et coll. $0=$ le présent travail.

là l'explication du désaccord entre nos résultats et ceux de la deuxième expérience de Welsh et coll. (vraisemblablement en raison d'une absorption sélective, d'une réflexion en fond de cellule et d'un manque de précision dans l'évaluation de la température).

V. Conclusion. - L'appareillage décrit a permis d'obtenir des résultats dans un domaine de pression et de température élevées. Il peut, moyennant des aménagements raisonnables, être adapté à l'étude d'autres vapeurs pures ou en mélange, dans un vaste domaine spectral.

L'extension du domaine de température est en cours et l'enceinte utilisée permet de travailler jusqu'à des pressions de l'ordre de 2000 bar. La présentation complète des phénomènes observés ainsi que les résultats obtenus par l'interprétation en cours seront donnés dans une publication ultérieure.

VI. Remerciements. - Nous tenons à remercier tout particulièrement Monsieur Ravel pour la réalisation des cellules de silice.

\section{Bibliographie}

[1] Leycuras (A.), Leycuras (Y.), Vodar (B.), $C . R$. Acad. Sci. B. Paris, 1966, 263, 885.

[2] Kikoin (I. K.) et Senchenkov (A. P.), Physics of Metals and metallography, 1967, 24, 74.

[3] Hensel (F.), Franck (E. U.), Ber. Bunsenges. Phys. Chem., 1966, 70, 1154.

[4] Schmutzler (R.), Hensel (F.), Phys. Letters, 1971, $35 \mathrm{~A}, 55$.

[5] Galt (J. A.) et Welsh (H. L.), Canadian Journal of Physics, 1957, 35, 98.

[6] Hensel (F.), Ber. Busenges. Phys. Chem., 1971, 75, 847.

[7] Wood (R. W.), Phil. Mag., 1909, 18, 187.

[8] Bender (J.), Physik Z., 1918, 19, 410.

[9] Welsh (H. L.), Kastner (J.) et Lauriston (A. C.), Canadian Journal of Research A, 1950, 28, 93.

[10] Granier (R.), Thèse, Ann. Phys., 1969, 4, 383.

[11] Tufeu (R.), Bouquet (M.), Le Neindre (B.), Mar-
TIN (G.), VoDAR (B.), High Temperatures, high pressures, 1970, 2, 351.

[12] Leycuras (A.), Leycuras (Y.), Vodar (B.), Traité des hautes températures, chapitre 9, Masson éditeur, 1972.

[13] Malbrunot (P.), Meunier (P.), Vidal (D.), High temperatures, high pressures, 1969, 1, 93.

[14] Sugawara (S.), Sato (T.) et Minamiyama (T.), Bulletin of JSME, 1962, 5, 711.

[15] Wood (R. W.) et Voss (V.), Proc. Roy. Soc. (London), A, 1928, 119, 698.

[16] Kuhn (H.) et Freudenberg (K.), Z. Physik, 1932, 76,38 .

[17] Kunn (H.), Proc. Roy. Soc. (London), A, 1937, 158, 212, 230.

[18] Mrozowski (S.), Phys. Rev., 1949, 76, 1714.

[19] UChTMANN (H.), Diplome, Université de Karlsruhe, 1970. 